\title{
EUNIS 2013: Unique IDM solutions at Lappeenranta University of Technology
}

\author{
Pekka Kuronen $^{1}$ \\ ${ }^{1}$ Lappeenranta, Finland \\ pekka.kuronen@lut.fi
}

Keywords

Identity Management, Identity and Access Management, IDM, IAM, Lappeenranta, University.

\section{ABSTRACT}

Lappeenranta University of Technology (LUT) is a dynamic, agile university setting special requirements for its Identity Management. We have developed unique solutions that have proven cost effective and time saving for our university and I believe would do so for other organizations as well.

At LUT we started implementing an IDM solution back in 2003 to meet the need for accurate login data across multiple systems. Since then we have ruled standard IDM solution models to be too inefficient for dynamic organization environments such as ours since it leaves lots of manual effort, coding and administration work in order to keep up with the environment.

From IDM point of view, LUT environment is a rapidly changing one with almost annually changing multiple identity sources, variable data syntaxes, new connected systems and constantly living organizational structures. These changes cannot be handled well with standard IDM implementation models.

Every solution is a compromise and we have had to do ones too but we have managed to pull it off quite well with some unique solutions. These solutions can handle dynamic situations with minimal effort keeping it simple as well.

Our solutions include:

- Contract based provisioning: a fine-grained and easy interface to identities. The use of contract based provisioning makes identity data input from multiple parallel sources very straightforward and easy to implement.

- Internal, automated auditing: autonomous auditing and resynchronizing of identity data. Regular internal data checking detects and repairs invalid data caused by event based operation with occasional faults in source systems, data communications or hardware. Data consistency is regained though triggering of the shared use of identity workflow policies.

- Dynamic organization tree: identities are stored in a tree that is updated in real time. Organizational tree is maintained in real time through standardized internal data format for organizational data enabling easy interface for organizational data from multiple sources.

- Centralized identity vault: all identity data is stored in our central identity vault. Centralized identity vault enables easy migration of new systems. The vault contains all the identity data available from source systems and calculated data that are refined suitable for current and future target systems.

- Three stage IDM model: the technical process is split into parts for easier configuration and less mistakes

I will try to open up these techniques, their requirements and benefits with my presentation at EUNIS 2013.

\section{REFERENCES}

NetIQ Identity Manager 4.0.2 documentation (2013). Retrieved May 2013, from: https://www.netiq.com/documentation/idm402/\#admin 


\section{AUTHOR'S BIOGRAPHY}

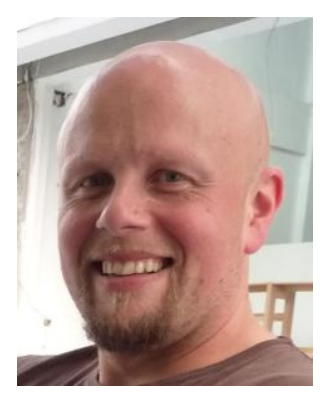

Contact data:

Pekka Kuronen

Huuhtakatu 7

53840 Lappeenranta

pekka.kuronen@lut.fi

+358405007099
Author is a former student of Lappeenranta University of Technology and an IT professional who has been dedicated to enterprise IT since 1995.

Most notable working experience includes:

- ISP services since 1995, founder of ISP company, administration of services in Lappeenranta University of Technology

- IDM/IAM since 2003. Design and implementation of multiple universities' IDM / IAM solutions.

Author is currently working as an employee at Lappeenranta University of Technology and as a private consultant. 